\title{
Improved Tumor Targeting of Anti-HER2 Nanobody Through $N$-Succinimidyl 4-Guanidinomethyl-3-Iodobenzoate Radiolabeling
}

\author{
Marek Pruszynski ${ }^{1}$, Eftychia Koumarianou$^{1}$, Ganesan Vaidyanathan $^{1}$, Hilde Revets $^{2}$, Nick Devoogdt ${ }^{3}$, Tony Lahoutte ${ }^{3}$, \\ H. Kim Lyerly ${ }^{4}$, and Michael R. Zalutsky ${ }^{1,5}$ \\ ${ }^{1}$ Department of Radiology, Duke University Medical Center, Durham, North Carolina; ${ }^{2}$ Ablynx N.V., Ghent, Belgium; ${ }^{3}$ In Vivo \\ Cellular and Molecular Imaging, Vrije Universiteit Brussel, Brussels, Belgium; ${ }^{4}$ Department of Surgery, Duke University Medical \\ Center, Durham, North Carolina; and ${ }^{5}$ Department of Radiation Oncology, Duke University Medical Center, Durham, North Carolina
}

Nanobodies are approximately $15-\mathrm{kDa}$ proteins based on the smallest functional fragments of naturally occurring heavy chainonly antibodies and represent an attractive platform for the development of molecularly targeted agents for cancer diagnosis and therapy. Because the human epidermal growth factor receptor type 2 (HER2) is overexpressed in breast and ovarian carcinoma, as well as in other malignancies, HER2-specific Nanobodies may be valuable radiodiagnostics and therapeutics for these diseases. The aim of the present study was to evaluate the tumor-targeting potential of antiHER2 5F7GGC Nanobody after radioiodination with the residualizing agent $N$-succinimidyl 4-guanidinomethyl 3-125/131/-iodobenzoate (*I-SGMIB). Methods: The 5F7GGC Nanobody was radiolabeled using *I-SGMIB and, for comparison, with $N^{\epsilon}-\left(3-{ }^{*} \mid-\right.$-iodobenzoyl)-Lys ${ }^{5}-N^{a_{-}}$ maleimido-Gly ${ }^{1}$-GEEEK ( ${ }^{*}$-IB-Mal-D-GEEEK), another residualizing agent, and by direct radioiodination using IODO-GEN ( ${ }^{125}$ I-Nanobody). The 3 labeled Nanobodies were evaluated in affinity measurements, and paired-label internalization assays were performed on HER2expressing BT474M1 breast carcinoma cells and in paired-label tissue distribution measurements in mice bearing subcutaneous BT474M1 xenografts. Results: *I-SGMIB-Nanobody was produced in $50.4 \% \pm 3.6 \%$ radiochemical yield and exhibited a dissociation constant of $1.5 \pm 0.5 \mathrm{nM}$. Internalization assays demonstrated that intracellular retention of radioactivity was up to 1.5-fold higher for *I-SGMIB-Nanobody than for coincubated ${ }^{125}$ I-Nanobody or *I-IBMal-D-GEEEK-Nanobody. Peak tumor uptake for *I-SGMIBNanobody was $24.50 \% \pm 9.89 \%$ injected dose/g at $2 \mathrm{~h}, 2$ - to 4 -fold higher than observed with other labeling methods, and was reduced by $90 \%$ with trastuzumab blocking, confirming the HER2 specificity of localization. Moreover, normal-organ clearance was fastest for *I-SGMIB-Nanobody, such that tumor-to-normal-organ ratios greater than 50:1 were reached by $24 \mathrm{~h}$ in all tissues except lungs and kidneys, for which the values were $10.4 \pm 4.5$ and $5.2 \pm$ 1.5, respectively. Conclusion: Labeling anti-HER2 Nanobody 5 F7GGC with *I-SGMIB yields a promising new conjugate for targeting HER2-expressing malignancies. Further research is needed to determine the potential utility of *I-SGMIB-5F7GGC labeled with ${ }^{124} \mid,{ }^{123}$, and ${ }^{131} \mid$ for PET and SPECT imaging and for targeted radiotherapy, respectively.

Received Jun. 3, 2013; revision accepted Nov. 19, 2013.

For correspondence or reprints contact: Michael R. Zalutsky, Department of Radiology, Box 3808, Duke University Medical Center, Durham, NC 27710. E-mail: zalut001@mc.duke.edu

Published online Feb. 27, 2014.

COPYRIGHT (C) 2014 by the Society of Nuclear Medicine and Molecular Imaging, Inc.
Key Words: HER2; Nanobody; breast cancer; radioiodination; SGMIB

J Nucl Med 2014; 55:650-656

DOI: 10.2967/jnumed.113.127100

$\mathbf{H}$ uman epidermal growth factor receptor type 2 (HER2) is overexpressed in $20 \%-30 \%$ of breast cancers and in a similar subset of gastric cancers, non-small cell lung cancers, colon carcinomas, and ovarian carcinomas and portends a poor prognosis (1-3). Novel drugs targeting HER2, including the monoclonal antibodies (mAbs) trastuzumab and pertuzumab, and tyrosine kinase inhibitors such as lapatinib, have been developed and shown significant therapeutic benefit in patients with HER2-expressing malignancies (4-6). Moreover, trastuzumab and pertuzumab have been evaluated in preclinical studies as potential targeting vectors for radioimmunotherapy $(7,8)$. Although radiolabeled HER2-specific constructs are of great interest not only for selecting patients for HER2-targeted therapies but also as targeted therapeutics, intact $\mathrm{mAbs}$ are not ideal vectors for either application because of their slow normal-tissue clearance. To overcome this drawback, a variety of small HER2-binding proteins have been developed, including scFv$\mathrm{C}_{\mathrm{H}} 2-\mathrm{C}_{\mathrm{H}} 3$ fragments (9), Affibody molecules (Affibody AB) (10) and their 2-helix analogs (11), and Nanobodies (variable fragments of heavy chain antibodies; Ablynx) (12).

Nanobodies are 12- to $15-\mathrm{kDa}$ nonimmunogenic antigen-binding single-domain fragments isolated from the heavy-chain-only antibodies occurring in Camelidae (13) with attractive features for radiolabeled imaging and therapy applications. Because they are small, they clear rapidly from blood and normal tissues and rapidly penetrate tumors. Moreover, they have better stability and solubility than whole mAbs, and they are able to recognize hidden or uncommon epitopes (14). Several Nanobodies with high affinities to HER2 have been developed $(12,15,16)$, with encouraging results providing motivation for further evaluation. Iodine radionuclides are appealing for this purpose because of the availability of radionuclides with decay characteristics suitable for SPECT $\left({ }^{123} \mathrm{I}\right)$ or PET $\left({ }^{124} \mathrm{I}\right)$ imaging as well as $\beta$-particle $\left({ }^{131} \mathrm{I}\right)$ or Auger electron $\left({ }^{123} \mathrm{I},{ }^{125} \mathrm{I}\right)$ radiotherapy.

An important consideration for the design of Nanobody-based radiopharmaceuticals is elucidation of radiolabeling strategies that 
will optimize their potential as imaging agents and therapeutics, both in terms of their general properties and in terms of features that are dependent on their interaction with targeted cells. With regard to the first consideration, excessive accumulation of radioactivity in the kidneys may be problematic (17) because Nanobodies are below the size cutoff for renal filtration. With regard to the second, HER2targeted proteins rapidly internalize after receptor binding $(7,8)$, a process that can lead to lysosomal degradation followed by rapid loss of radioactivity from tumor cells $(18,19)$.

This consideration has led to the development of prosthetic groups that can be conjugated to internalizing mAbs so that labeled catabolites generated from the conjugate are trapped within the cell. Two strategies that we have explored for designing residualizing agents are the use of aromatic acylation agents bearing substituents that will remain charged at lysosomal $\mathrm{pH}(20,21)$ and short D-amino acid peptides that are inert to lysosomal hydrolases $(22,23)$. Because $N^{\epsilon}$-(3-iodobenzoyl)-Lys ${ }^{5}$ - $N^{\alpha}$-maleimido-Gly ${ }^{1}$-GEEEK (IB-Mal-DGEEEK) was particularly effective in enhancing tumor retention of radioactivity for internalizing $\mathrm{mAbs}$ and fragments $(18,23)$, this reagent was selected for initial Nanobody labeling studies. Although IB-Mal-D-GEEEK offered significant advantages over conventional iodination (16), better tumor targeting should be achievable with alternative labeling approaches.

In the present study, the anti-HER2 Nanobody 5F7GGC was radioiodinated using $\mathrm{N}$-succinimidyl 4-guanidinomethyl-3-iodobenzoate (SGMIB) - a residualizing agent that combines good tumor retention and rapid normal-tissue clearance (18) - and was evaluated for targeting HER2-expressing BT474M1 human breast carcinoma cells and xenografts. Paired-label comparisons were made to Nanobody labeled using IB-Mal-D-GEEEK and 1,3,4,6-tetrachloro-3 $\alpha, 6 \alpha$ diphenyl-glycoluril (IODO-GEN; Thermo Fisher Scientific).

\section{MATERIALS AND METHODS}

\section{Nanobody Molecule}

Production, purification, and characterization of 5F7GGC Nanobody bearing a cysteine tail have been described elsewhere (16). Briefly, the anti-HER2 Nanobody was identified from phage libraries derived from llamas immunized with SKBR3 human breast carcinoma cells. Its equilibrium dissociation constant for binding to HER2, determined by surface plasmon resonance, was $0.51 \mathrm{nM}$.

\section{Nanobody Radioiodination}

Details for the synthesis and high-performance liquid chromatography purification of ${ }^{125 / 131}$ I-SGMIB and ${ }^{125 / 131}$ I-IB-Mal-D-GEEEK from 4-( $\left(N^{1}, N^{2}\right.$-bis-tert-butyloxycarbonyl)guanidinomethyl-3-trimethylstannylbenzoate and $N^{\epsilon}$-(3-(tri-n-butyl)benzoyl)-Lys ${ }^{5}-N^{\alpha}$-maleimido-Gly ${ }^{1}$ D-GEEEK, respectively, and methods for their conjugation to proteins are summarized in the Supplemental Materials (available at http://jnm. snmjournals.org) and described in detail elsewhere $(23,24)$. The 5 F7GGC Nanobody was radiolabeled on constituent tyrosine residues using the IODO-GEN method (16). Aliquots of $96 \mu \mathrm{g}$ of 5F7GGC Nanobody were used for all labeling procedures. Nanobody labeled using IODO-GEN (*I-Nanobody; $* \mathrm{I}={ }^{125} \mathrm{I}$ or ${ }^{131} \mathrm{I}$ ), SGMIB (*ISGMIB-Nanobody), and IB-Mal-D-GEEEK (*I-IB-Mal-D-GEEEKNanobody) were purified by gel filtration over PD-10 columns (GE Healthcare) eluted with phosphate-buffered saline, $\mathrm{pH}$ 7.4. Proteinassociated radioactivity was determined by instant thin-layer chromatography and coprecipitation with human serum albumin using $20 \%$ trichloroacetic acid. The immunoreactivity of radiolabeled Nanobodies was determined by Lindmo assay using magnetic beads coated with recombinant ErbB2/HER2 Fc chimera (R\&D Systems) or bovine serum albumin to correct for nonspecific binding $(16,22)$.

\section{Binding Affinity and Internalization Assays}

These studies were performed using HER2-expressing BT474M1 human breast carcinoma cells (25). Cells were grown in Dulbecco modified Eagle/F12 medium supplemented with $10 \%$ fetal calf serum, streptomycin $(100 \mu \mathrm{g} / \mathrm{mL}$ ), and penicillin (100 IU/mL) (Sigma Aldrich) at $37^{\circ} \mathrm{C}$ in $5 \% \mathrm{CO}_{2}$. Medium was changed every $2 \mathrm{~d}$ and cells passaged by trypsinization $(0.05 \%$ trypsin-ethylenediamine tetraacetic acid) when they were about $80 \%$ confluent. Binding affinities were determined by incubation of $0.1-300 \mathrm{nM}$ labeled Nanobodies with BT474M1 cells as described previously (16), and equilibrium dissociation constants were determined using GraphPad Prism software. Internalization and cell processing assays involved incubation of 7.7 pmol of labeled Nanobodies with $8 \times 10^{4}$ BT474M1 cells at $4^{\circ} \mathrm{C}$ for $30 \mathrm{~min}$, removal of unbound Nanobody, and then incubation at $37^{\circ} \mathrm{C}$ for $24 \mathrm{~h}$, as described previously (16). Nonspecific uptake was determined by coincubation with a 100 -fold excess of trastuzumab (Genentech).

\section{Paired-Label Biodistribution Studies}

Animal studies were performed in accordance with the guidelines established by the Duke University Institutional Animal Care and Use Committee. Sixty-day continuous-release $17-\beta$-estradiol pellets (Innovative Research of America) were implanted in the backs of 10- to 12wk-old female NOD.CB17-Prkdc scid/J mice (Jackson Laboratories). Two days later, the mice were inoculated in the flank with $5 \times 10^{6}$ BT474M1 cells in 50\% Matrigel (BD Biosciences). Biodistribution studies were initiated when tumors reached a volume of $350-500 \mathrm{~mm}^{3}$.

Five groups of 5 mice were injected via the tail vein with $185 \mathrm{kBq}$ of both ${ }^{125} \mathrm{I}$-Nanobody $(0.6 \mu \mathrm{g}, 294 \mathrm{MBq} / \mathrm{mg})$ and ${ }^{131} \mathrm{I}$-SGMIB-Nanobody $(1.0 \mu \mathrm{g}, 185 \mathrm{MBq} / \mathrm{mg})$ or $93 \mathrm{kBq}$ of ${ }^{125} \mathrm{I}-\mathrm{SGMIB}-$ Nanobody $(1.6 \mu \mathrm{g}$, $59 \mathrm{MBq} / \mathrm{mg}$ ) and $150 \mathrm{kBq}$ of ${ }^{131}$ I-IB-Mal-D-GEEEK-Nanobody $(0.7 \mu \mathrm{g}, 218 \mathrm{MBq} / \mathrm{mg})$. At 1, 2, 4, 8, and $24 \mathrm{~h}$ after injection, the mice were euthanized by halothane overdose, dissected, and their organs collected. An additional group of 5 mice in each experiment was injected with trastuzumab ( $\sim 500$-fold molar excess $) 24 \mathrm{~h}$ before labeled Nanobody administration, and $2 \mathrm{~h}$ later, their biodistribution was determined. Tissues of interest were weighed and counted in a dual-channel $\gamma$ counter for ${ }^{125} \mathrm{I}$ and ${ }^{131} \mathrm{I}$ radioactivity along with injection standards. Results were expressed as percentage injected dose per gram of tissue (\%ID/g), except for thyroid, for which \%ID/organ values were calculated.

\section{Statistical Analyses}

Data are presented as mean \pm SD. Differences in tissue uptake of coadministered labeled Nanobodies were tested for statistical significance with a paired 2-tailed Student $t$ test using Microsoft Excel. A $P$ value of less than 0.05 was considered statistically significant.

\section{RESULTS}

\section{Radiolabeling}

The radioiodination yield for labeling 5F7GGC Nanobody using the IODO-GEN, *I-SGMIB, and *I-IB-Mal-D-GEEEK methods was $86.2 \% \pm 1.6 \%(n=5), 50.4 \% \pm 3.6 \%(n=3)$, and $69.6 \% \pm$ $5.6 \%(n=6)$, respectively, and radiochemical purity was greater than $98 \%$ with each method. Specific activities of $118-910 \mathrm{MBq} / \mathrm{mg}$, 59-160 MBq/mg, and 22-352 MBq/mg were obtained for Nanobodies labeled using IODO-GEN, *I-SGMIB, and *I-IB-Mal-D-GEEEK, respectively. Immunoreactive fractions for *I-Nanobody, *I-SGMIBNanobody, and *I-IB-Mal-D-GEEEK-Nanobody binding to HER2 were $59.5 \% \pm 3.9 \%(n=3), 70.4 \% \pm 15.7 \%(n=3)$, and $74.6 \% \pm$ $18.5 \%(n=5)$, respectively.

\section{Binding Affinity and Internalization}

Binding affinity was evaluated using the BT474M1 human breast carcinoma cell line. The equilibrium dissociation constant 
measured for ${ }^{125} \mathrm{I}$-SGMIB-Nanobody was $1.5 \pm 0.5 \mathrm{nM}$ (Supplemental Fig. 1), a value similar to values reported previously for ${ }^{125} \mathrm{I}-$ Nanobody $(1.8 \pm 0.6 \mathrm{nM})$ and ${ }^{131}$ I-IB-Mal-D-GEEEK-Nanobody $(3.2 \pm 1.0 \mathrm{nM})(16)$.

Two assays were performed to directly compare the intracellular retention of radioactivity in BT474M1 cells of *I-SGMIB-Nanobody with that of coincubated ${ }^{125}$ I-Nanobody or ${ }^{131}$ I-IB-Mal-D-GEEEKNanobody (Fig. 1). In the first study, intracellular counts from ${ }^{125} \mathrm{I}-$ Nanobody $(68.8 \% \pm 6.2 \%)$ and ${ }^{131}$ I-SGMIB-Nanobody $(73.8 \% \pm$ $1.3 \%$ ) of initially cell-bound activity were similar after $1 \mathrm{~h}$ and steadily decreased with time for ${ }^{125} \mathrm{I}-\mathrm{Nanobody}$, reaching $36.6 \% \pm$ $4.1 \%$ at $24 \mathrm{~h}$. In contrast, intracellular radioactivity from ${ }^{131} \mathrm{I}-$ SGMIB-Nanobody remained fairly constant and was $57.6 \% \pm$ $6.3 \%$ at $24 \mathrm{~h}$. Direct comparison of the internalization of ${ }^{125} \mathrm{I}-$ SGMIB-Nanobody and ${ }^{131}$ I-IB-Mal-D-GEEEK-Nanobody revealed that the intracellular radioactivity from ${ }^{131}$ I-IB-Mal-D-GEEEKNanobody was constant over $24 \mathrm{~h}(46.8 \% \pm 13.3 \%$ at $1 \mathrm{~h} ; 48.2 \%$ $\pm 1.7 \%$ at $24 \mathrm{~h}$ ), whereas internalized counts from ${ }^{125}$ I-SGMIBNanobody slightly decreased with time $(64.3 \% \pm 11.6 \%$ at $1 \mathrm{~h}$; $52.0 \% \pm 2.4 \%$ at $24 \mathrm{~h}$ ). Intracellular activity for ${ }^{125}$ I-SGMIB-Nanobody was higher than that from ${ }^{131}$ I-IB-Mal-D-GEEEK-Nanobody at all time points, with the differences being statistically significant at 4 and $8 \mathrm{~h}(P<0.05)$. As expected, complementary behavior was observed in cell culture supernatant activity levels, consistent with release of labeled catabolites into the medium. Pretreatment of BT474M1 cells with a 100-fold excess of trastuzumab reduced intracellular radioactivity to less than $0.2 \%$, demonstrating the HER2 specificity of labeled Nanobody internalization. A significantly higher fraction of cell culture supernatant activity was protein-associated for ${ }^{131}$ I-SGMIB-Nanobody than for ${ }^{125}$ I-Nanobody $(P<0.05)$ at all time points. Protein-associated activity for ${ }^{125}$ I-SGMIB-Nanobody and

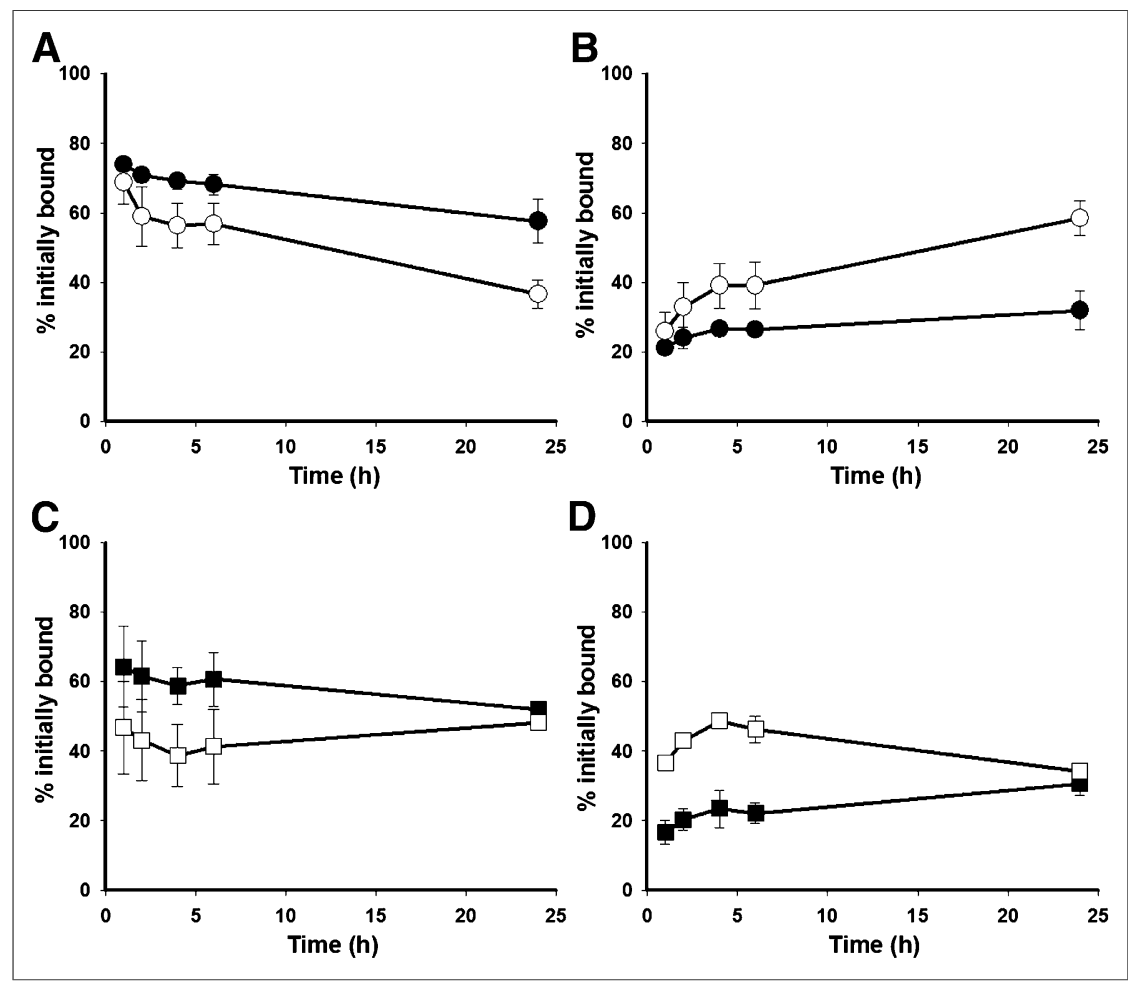

FIGURE 1. Cellular processing of radioiodinated Nanobody in BT474M1 cells. (A and B) ${ }^{125}$ Nanobody $(O)$ vs. ${ }^{131}$ I-SGMIB-Nanobody $(\bullet)$ : internalized $(A)$ and supernatant $(B)$. (C and D) ${ }^{131}$ IIB-Mal-D-GEEEK-Nanobody ( $\square$ ) vs. ${ }^{125}$ I-SGMIB-Nanobody ( $(\square)$ : internalized (C) and supernatant (D).
${ }^{131}$ I-IB-Mal-D-GEEEK-Nanobody was $86 \%-95 \%$ over the first $6 \mathrm{~h}$ (differences not significant); however, at $24 \mathrm{~h}$, trichloroacetic acidprecipitable activity for ${ }^{125}$ I-SGMIB-Nanobody decreased to $43.1 \% \pm 0.6 \%$ whereas that for ${ }^{131}$ I-IB-Mal-D-GEEEK-Nanobody was $82.2 \% \pm 7.2 \%$.

\section{Biodistribution Studies}

The tissue distribution of *I-SGMIB-Nanobody was compared with ${ }^{125}$ I-Nanobody and ${ }^{131}$ I-IB-Mal-D-GEEEK-Nanobody in mice bearing BT474M1 xenografts, and the results in all tissues obtained 1-24 $\mathrm{h}$ after injection are presented in Supplemental Tables 1 and 2, respectively. The most striking differences were observed in tumor and kidneys (Fig. 2). Tumor uptake of ${ }^{131} \mathrm{I}-$ SGMIB-Nanobody was significantly higher than that of ${ }^{125} \mathrm{I}$ Nanobody at all time points, peaking at $24.50 \pm 9.89 \%$ ID/g after $2 \mathrm{~h}$, compared with $6.39 \pm 1.97 \% \mathrm{ID} / \mathrm{g}$ for ${ }^{125} \mathrm{I}-\mathrm{Nanobody}$, with the tumor delivery advantage for ${ }^{131} \mathrm{I}$-SGMIB-Nanobody reaching nearly 8 -fold at $24 \mathrm{~h}$. The average tumor weight at necropsy was $0.31 \pm 0.07 \mathrm{~g}$. Renal uptake of ${ }^{131}$ I-SGMIB-Nanobody was significantly higher than that of ${ }^{125} \mathrm{I}-\mathrm{Nanobody}$ at 1 and $2 \mathrm{~h}$; however, by $24 \mathrm{~h},{ }^{131}$ I-SGMIB-Nanobody exhibited 5-fold lower kidney uptake than ${ }^{125}$ I-Nanobody $(P<0.004)$. Tumor-to-normal-tissue ratios were significantly higher for ${ }^{131}$ I-SGMIB-Nanobody than for ${ }^{125}$ I-Nanobody (Supplemental Fig. 2). For example, tumor-to-blood and tumor-to-muscle ratios were $10.9 \pm 2.4$ and $18.8 \pm 8.9$, respectively, for ${ }^{131} \mathrm{I}$-SGMIB-Nanobody at $1 \mathrm{~h}$, compared with $0.5 \pm 0.1$ and $4.2 \pm 1.1$ for ${ }^{125} \mathrm{I}$-Nanobody.

In the second experiment, tumor accumulation peaked at $2 \mathrm{~h}$ for ${ }^{125} \mathrm{I}$-SGMIB-Nanobody $(12.57 \pm 2.77 \% \mathrm{ID} / \mathrm{g})$ and ${ }^{131} \mathrm{I}$-IB-Mal-DGEEEK-Nanobody $(6.23 \pm 1.32 \% \mathrm{ID} / \mathrm{g})$, with an approximately 2-fold tumor delivery advantage maintained throughout $24 \mathrm{~h}$ (Fig. 2C). The average tumor weight at necropsy was $0.72 \pm 0.29 \mathrm{~g}$. Uptake of ${ }^{125}$ I-SGMIB-Nanobody in the kidney was 2-fold lower than that of coadministered ${ }^{131}$ I-IB-Mal-D-GEEEK-Nanobody at $1 \mathrm{~h}$ $(82.4 \pm 15.3 \% \mathrm{ID} / \mathrm{g}$ vs. $196.6 \pm 23.9 \%$ $\mathrm{ID} / \mathrm{g}$ ) and 50-fold lower at $24 \mathrm{~h}$. Thyroid and stomach radioiodine levels for Nanobody labeled with either *I-SGMIB or *IIB-Mal-D-GEEEK were 20 - to $\geq 200$-fold lower that those seen for directly radioiodinated Nanobody (Supplemental Tables 1 and 2), consistent with a low degree of deiodination in vivo for Nanobody labeled using these residualizing agents. Tumorto-normal-tissue ratios were higher for ${ }^{125}$ I-SGMIB-Nanobody than for ${ }^{131}$ I-IBMal-D-GEEEK-Nanobody, especially at later time points (Fig. 3). For example, tumor-to-blood ratios for ${ }^{125}$ I-SGMIBNanobody increased from $8.2 \pm 3.3$ to $36.4 \pm 6.9$ from 1 to $4 \mathrm{~h}$, compared with $4.3 \pm 1.5$ to $16.5 \pm 3.9$ for ${ }^{131}$ I-IB-MalD-GEEEK-Nanobody. Tumor-to-normaltissue ratios for ${ }^{125} \mathrm{I}$-SGMIB-Nanobody were at least 10 at $8 \mathrm{~h}$ for all tissues except kidneys, for which a value of $1.9 \pm 0.8$, compared with $0.04 \pm 0.01$ for ${ }^{131}$ I-IB-Mal-D-GEEEK-Nanobody, was observed. 


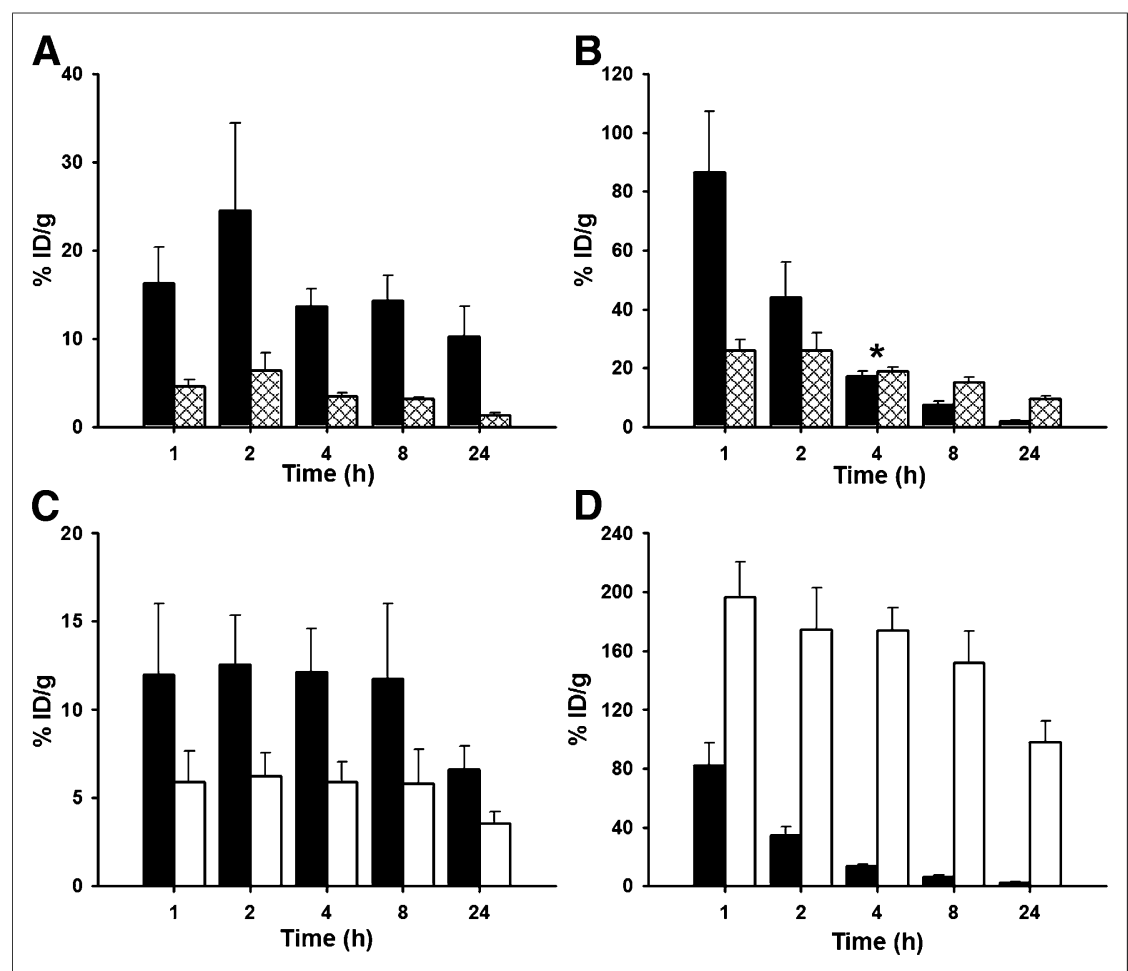

FIGURE 2. Uptake of radioiodine in athymic mice with BT474M1 xenografts. (A and B) ${ }^{125}$ Nanobody (hatched) vs. ${ }^{131}$ I-SGMIB-Nanobody (black): tumor (A) and kidneys (B). (C and D) ${ }^{131}$ IIB-Mal-D-GEEEK-Nanobody (white) vs. ${ }^{125}$-SGMIB-Nanobody (black): tumor (C) and kidneys (D). ${ }^{*}$ Difference not significant $(P>0.05)$.

The effect of a blocking dose of trastuzumab administered $24 \mathrm{~h}$ before labeled Nanobodies was determined to evaluate the HER2 specificity of labeled Nanobody distribution (Table 1). Preinjection of trastuzumab reduced tumor uptake in the first study from $6.4 \% \pm 2.0 \%$ to $2.3 \% \pm 0.2 \%$ for ${ }^{125}$ I-Nanobody ( $65 \%$ inhibition) and from $24.5 \% \pm 9.9 \%$ to $2.1 \% \pm 0.2 \%$ for ${ }^{131}$ I-SGMIB-Nanobody ( $90 \%$ inhibition). In the second study, preinjection of trastuzumab reduced tumor uptake over $90 \%$ for both ${ }^{125}$ I-SGMIB-Nanobody and ${ }^{131}$ I-IB-Mal-D-GEEEK-Nanobody but did not affect uptake in other tissues, confirming HER2-specific tumor localization of these labeled Nanobodies.
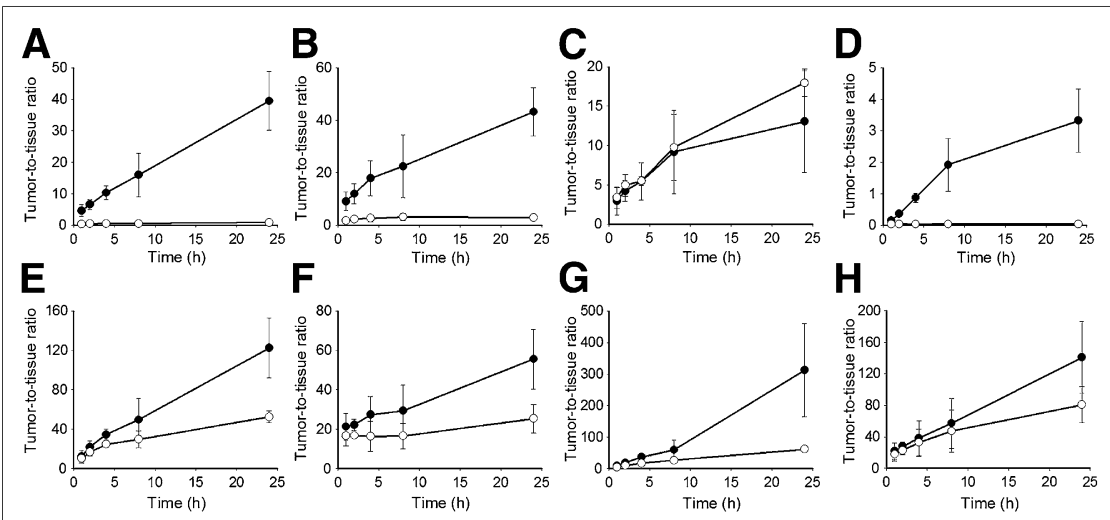

FIGURE 3. Tumor-to-normal-tissue ratios in athymic mice bearing subcutaneous BT474M1 xenografts for ${ }^{131}$ |-IB-Mal-D-GEEEK-Nanobody (O) and ${ }^{125}$ I-SGMIB-Nanobody (•): liver (A), spleen $(B)$, lungs $(C)$, kidneys $(D)$, heart $(E)$, stomach $(F)$, blood $(G)$, and muscle $(H)$.
Radiation dosimetry calculations were performed from these mouse distribution data for hypothetical 37-MBq doses of ${ }^{131} \mathrm{I}-$ labeled Nanobody according to methods described in the Supplemental Materials. The radiation dose delivered to tumor from ${ }^{131}$ I-SGMIB-Nanobody was about 2 times higher than that calculated for ${ }^{131}$ I-IB-MalD-GEEEK-Nanobody in the same animals; a greater than 6-fold tumor dose delivery advantage was estimated compared with ${ }^{131}$ I-Nanobody (Supplemental Table 3). Radiation dose delivered to kidney for ${ }^{131}$ I-SGMIB-Nanobody was about 2-fold lower than that to tumor, whereas the kidney radiation dose for ${ }^{131}$ I-IB-Mal-DGEEEK-Nanobody and ${ }^{131}$ I-Nanobody was 28 and 7 times higher, respectively. Tumor-to-normal-tissue radiation dose ratios for ${ }^{131}$ I-SGMIB-Nanobody were higher than those determined for the 2 other labeling methods and were about $32,47,8,56$, and 104 in liver, spleen, lungs, stomach, and blood, respectively.

\section{DISCUSSION}

Nanobodies are the smallest antigen recognition fragments derived from a functional $\mathrm{mAb}$, a fortuitous consequence of the heavy-chain-only mAb configuration occurring naturally in Camelidae. These approximately $15-\mathrm{kDa}$ proteins are an attractive platform for the development of molecularly specific radiopharmaceuticals, combining the rapid blood clearance and tumor penetration of similarly sized Affibody and DARPin molecules (designed ankyrin repeat proteins) $(10,26)$ with other potential advantages. These include the ability to recognize buried or hidden epitopes because of the conformation of their CDR3 domains and ease of generation of high-affinity binders compared with constructs derived from artificial protein scaffolds such as Affibody molecules (14). To harness the potential advantages of Nanobodies as radiodiagnostic and, possibly, radiotherapeutic agents, several challenges must be addressed, many of which are a consequence of their small size. First, compared with intact mAbs, the CDR domains, which are responsible for target recognition, comprise a greater fraction of the molecule, increasing the likelihood that radiolabeling will alter binding affinity. Second, because Nanobodies are below the size limit for renal filtration, achieving adequate tumor levels while minimizing retention in the kidneys will be important. Finally, for Nanobodies that bind to internalizing receptors such as HER2, a radionuclide-radiolabeling pair that results in efficient trapping of the radioactivity in tumor cells after intracellular processing of the labeled Nanobody should be exploited, particularly if therapeutic application is envisioned. 
TABLE 1

Effect of $10 \mathrm{mg}$ of Trastuzumab on Radioiodinated Nanobody Uptake in BT474M1 Xenografts and Normal Tissues

\begin{tabular}{|c|c|c|c|c|c|c|c|c|}
\hline \multirow[b]{3}{*}{ Tissue } & \multicolumn{4}{|c|}{ Experiment 1} & \multicolumn{4}{|c|}{ Experiment 2} \\
\hline & \multicolumn{2}{|c|}{$\begin{array}{c}\text { 125I-Nanobody (185 kBq, } \\
0.6 \mu \mathrm{g})\end{array}$} & \multicolumn{2}{|c|}{$\begin{array}{l}\text { 131/-SGMIB-Nanobody } \\
(185 \mathrm{kBq}, 1.0 \mu \mathrm{g})\end{array}$} & \multicolumn{2}{|c|}{$\begin{array}{l}\text { 125I-SGMIB-Nanobody } \\
(93 \mathrm{kBq}, 1.6 \mu \mathrm{g})\end{array}$} & \multicolumn{2}{|c|}{$\begin{array}{l}\text { 131/-IB-Mal-D-GEEK- } \\
\text { Nanobody }(150 \mathrm{kBq}, 0.7 \mu \mathrm{g})\end{array}$} \\
\hline & Not blocked & Blocked & Not blocked & Blocked & Not blocked & Blocked & Not blocked & Blocked \\
\hline Liver & $7.76 \pm 1.56$ & $6.28 \pm 0.72$ & $2.65 \pm 0.65$ & $2.45 \pm 0.34$ & $1.95 \pm 0.42$ & $1.72 \pm 0.11$ & $13.32 \pm 1.75$ & $12.71 \pm 0.76$ \\
\hline Spleen & $3.90 \pm 1.77$ & $3.32 \pm 0.49$ & $1.13 \pm 0.47$ & $1.03 \pm 0.37$ & $1.09 \pm 0.29$ & $0.92 \pm 0.14$ & $2.68 \pm 0.38$ & $2.99 \pm 0.86$ \\
\hline Kidneys & $26.05 \pm 5.86$ & $21.54 \pm 1.09$ & $44.0 \pm 12.0$ & $42.30 \pm 4.66$ & $34.98 \pm 5.57$ & $36.89 \pm 5.48$ & $174.8 \pm 28.2$ & $197.2 \pm 22.4$ \\
\hline Blood & $10.08 \pm 1.47$ & $6.56 \pm 0.65^{\star}$ & $1.15 \pm 0.25$ & $1.04 \pm 0.16$ & $0.70 \pm 0.10$ & $0.69 \pm 0.09$ & $0.69 \pm 0.09$ & $0.70 \pm 0.09$ \\
\hline Tumor & $6.39 \pm 1.97$ & $2.27 \pm 0.22^{*}$ & $24.50 \pm 9.89$ & $2.14 \pm 0.21^{*}$ & $12.57 \pm 2.77$ & $1.26 \pm 0.13^{*}$ & $6.23 \pm 1.32$ & $0.68 \pm 0.06^{\star}$ \\
\hline
\end{tabular}

*Significant blocking effect $(P<0.05)$.

Data are \%ID/g (mean $\pm \mathrm{SD} ; n=5)$.

Our initial studies with anti-HER2 Nanobody 5F7GGC established the need for using a residualizing labeling strategy for this rapidly internalizing construct (16). IB-Mal-D-GEEEK was selected for this purpose because of the excellent results obtained when this prosthetic group was used to label other internalizing mAbs and fragments. For example, labeling the antiepidermal growth factor variant III (EGFRvIII) mAb L8A4 increased retention of radioiodine in EGFRvIII-expressing cells and xenografts by factors of up to 15-fold and 5-fold, respectively, compared with L8A4 labeled using IODO-GEN (23). Although less striking advantages were observed, labeling a $105-\mathrm{kDa}$ anti-HER2 $\left(\mathrm{scFv}-\mathrm{C}_{\mathrm{H}} 2-\mathrm{C}_{\mathrm{H}} 3\right)_{2}$ with IB-Mal-D-GEEEK also resulted in a significant tumor delivery advantage compared with IODO-GEN or SGMIB labeling (18). With 5F7GGC Nanobody, IB-Mal-D-GEEEK labeling increased tumor uptake by 1.5 -fold at $8 \mathrm{~h}$ and 3 -fold at $24 \mathrm{~h}$ compared with IODOGEN (16). Unfortunately, IB-Mal-D-GEEEK labeling also resulted in more than an order of magnitude increase in kidney uptake to levels in excess of $100 \% \mathrm{ID} / \mathrm{g}$ at all time points, providing motivation for the current study, seeking a labeling approach that would enhance tumor retention without engendering an even greater increase in kidney uptake.

The residualizing property of IB-Mal-D-GEEEK is derived from the 3 negatively charged glutamic acid residues in the D-amino acid pentapeptide, which generate labeled catabolites that are trapped in tumor cells after receptor-mediated internalization of proteins labeled with this reagent (23). Exploiting the limited diffusion of labeled catabolites across lysosomal and cell membranes as a strategy for labeling internalizing proteins can also be accomplished using aromatic prosthetic groups bearing charged substituents. The SGMIB reagent exemplifies this tactic, with one positive charge created by a highly basic guanidine moiety (20). Although significant tumor enhancement was observed when anti-EGFRvIII mAb L8A4 was labeled using SGMIB, the effect was lower than that seen when IB-Mal-D-GEEEK was used to label this mAb. On the other hand, kidney retention of likely catabolites for proteins labeled using SGMIB were very low $(20,27)$, which is not the case for charged D-amino acid acylation agents (28), making SGMIB of potential value as a Nanobody-labeling reagent.

With only 5 lysines in the 5F7GGC molecule and none found in the CDRs (data not shown), the formation of a high-affinity SGMIB-Nanobody conjugate should be feasible. Indeed, the in vitro affinity measured for binding of ${ }^{125} \mathrm{I}$-SGMIB-Nanobody to BT474M1 cells was $1.5 \pm 0.5 \mathrm{nM}$, in good agreement with a value of $0.51 \mathrm{nM}$ for the binding of cold Nanobody to HER2 extracellular domain determined by surface plasmon resonance (16). Paired-label internalization and cell-processing assays demonstrated that intracellular retention of radioactivity for *I-SGMIBNanobody was higher than that for ${ }^{125}$ I-Nanobody at all time points and higher than that for ${ }^{131} \mathrm{I}-\mathrm{IB}-\mathrm{Mal}-\mathrm{D}-\mathrm{GEEEK}-$ Nanobody at all time points except $24 \mathrm{~h}$. Internalized counts for Nanobody labeled using IB-Mal-D-GEEEK appear to be increasing with time, an observation that has been seen to a greater extent when other receptor-targeted proteins have been labeled with this reagent but not with other labeling methods $(18,23)$. The fact that proteinassociated activity in the cell culture supernatant was highest for ${ }^{131}$ I-IB-Mal-D-GEEEK-Nanobody than for the 2 other labeled Nanobodies at $24 \mathrm{~h}$ is consistent with this observation. Although the reasons for this behavior are not clear, one possibility is that differences in recycling of the receptor-Nanobody complex or its susceptibility to degradation could play a role.

Results from paired-label studies in SCID mice bearing BT474M1 xenografts demonstrated superior biodistribution and tumor targeting for the SGMIB-Nanobody conjugate. The tumor uptake obtained with *I-SGMIB-Nanobody was 4-8 times higher than with coadministered ${ }^{125}$ I-Nanobody and about 2 times higher than with coadministered ${ }^{131}$ I-IB-Mal-D-GEEEK-Nanobody at all time points. The tumor accumulation of *I-SGMIB-Nanobody peaked at $24.50 \pm 9.89$ and $12.57 \pm 2.77 \% \mathrm{ID} / \mathrm{g}$ in the 2 experiments, with the difference likely reflecting the approximately 2 -fold larger size of the xenografts in the second study (29). Although comparisons to results obtained in other xenograft models must be done with caution, it is encouraging to note that the magnitude of tumor accumulation observed with *I-SGMIB-Nanobody also was 2-4 times higher than reported for other anti-HER2-targeted Nanobodies labeled with ${ }^{99 \mathrm{~m}} \mathrm{Tc}(12),{ }^{177} \mathrm{Lu}(15)$, and ${ }^{68} \mathrm{Ga}(30)$, as well as ${ }^{99 \mathrm{~m}}$ Tc-labeled (17) and ${ }^{68} \mathrm{Ga}$-labeled (31) Nanobodies targeting EGFR. Moreover, *I-SGMIB-Nanobody exhibits a HER2 xenograft delivery advantage similar to that of other HER2-targeted proteins, including Affibody molecules labeled with ${ }^{18} \mathrm{~F},{ }^{68} \mathrm{Ga}$, and ${ }^{111}$ In (32).

Perhaps the most significant limitation of Nanobodies as molecular carriers for radionuclides is that they can result in high radioactivity levels in the kidneys. This characteristic is commonly observed for proteins with molecular weights of less than $60 \mathrm{kDa}$ and can be attributed to renal tubular reabsorption (33). Another advantage of *I-SGMIB-Nanobody for targeting radionuclides to 
HER2-expressing tumors is that unlike *I-IB-Mal-D-GEEEKNanobody, enhancement of tumor uptake compared with directly labeled Nanobody was achieved without a substantially greater increase in kidney radioactivity levels. Moreover, with *I-SGMIBNanobody, radioactivity cleared from the kidney much more rapidly than with the other 2 radioiodinated Nanobody conjugates, with a half-life of about $1 \mathrm{~h}$. By $8 \mathrm{~h}$ after injection, the tumor-to-kidney ratio for *I-SGMIB-Nanobody was $2.0 \pm 0.5$, compared with $0.21 \pm 0.02$ and $0.04 \pm 0.01$ for ${ }^{125}$ I-Nanobody and ${ }^{131}$ I-IB-MalD-GEEEK-Nanobody, respectively. In addition, retention of $*$ ISGMIB-Nanobody in the kidney was lower than that reported for eleven ${ }^{99 \mathrm{~m}}$ Tc-labeled anti-HER2 Nanobodies (12), as well as an anti-HER2 Affibody labeled with ${ }^{18} \mathrm{~F},{ }^{68} \mathrm{Ga}$, and ${ }^{111} \mathrm{In}(32)$.

In interpreting the renal uptake of labeled Nanobodies, it is important to bear in mind that in addition to protein size, one must also consider the effects of labeling method and radionuclide on kidney radioactivity levels. In the current study, renal activity levels for Nanobody labeled using the 2 residualizing labeling methodsSGMIB and IB-Mal-D-GEEEK - were significantly higher than those seen for directly labeled Nanobody at early time points. Although dehalogenation of ${ }^{125}$ I-Nanobody followed by escape of radioiodide from the kidney could have played a role, residualizing labels can be trapped in the lysosomes of not only tumor cells but also other cells that catabolize proteins, including liver, spleen, and kidney (34). In the current study, the renal uptake, and to a lesser degree, the uptake in spleen and liver, of *I-IB-Mal-D-GEEEK-Nanobody was considerably higher than observed with *I-SGMIB-Nanobody, suggesting that the nature of the residualizing agent has a significant influence on radioactivity levels in these tissues.

Radioactivity levels in kidney, liver, and spleen with ${ }^{131} \mathrm{I}-\mathrm{IB}-$ Mal-D-GEEEK-Nanobody remained almost constant during the first $8 \mathrm{~h}$, whereas initial activity levels in these organs were lower and cleared more quickly with *I-SGMIB-Nanobody. These results are consistent with the results obtained when an antiHER2 ( $\left.\mathrm{scFv}-\mathrm{C}_{\mathrm{H}} 2-\mathrm{C}_{\mathrm{H}} 3\right)_{2}$ was radioiodinated by these 2 methods (18). The higher levels of radioactivity in these organs from ${ }^{131} \mathrm{I}-$ IB-Mal-D-GEEEK-Nanobody cannot be attributed to a stronger residualizing effect of IB-Mal-D-GEEEK because the same effect was not seen in tumor. The significantly improved kidney uptake and clearance characteristics of *I-SGMIB-Nanobody compared with *I-IB-Mal-D-GEEEK-Nanobody likely reflect differences in the nature of catabolites generated in vivo with these 2 labeling approaches. In previous studies, it was found that the primary catabolite of mAbs labeled using SGMIB is a conjugate of 4guanidinomethyl-3-iodobenzoic acid and glycine, which is known to be excreted rapidly into the urine $(20,27)$. With IB-Mal-DGEEEK, the D-amino acid pentapeptide is expected to be the catabolite because its peptide bonds should be resistant to proteolytic cleavage $(28,35)$. High kidney uptake of several labeled molecules containing glutamic acid residues has been reported $(36,37)$ and has been attributed to the presence of several amino acid transport systems in mammalian kidney, especially EAAT3, which is one of the major transporters for L-glutamic acid (38). In addition, even if the above-mentioned transporters are stereospecific, Schuldt et al. (39) have reported the existence of a transporter at the antiluminal surface of renal tubule cells that could be accountable for D-glutamate uptake. Finally, differences in polarity between the labeled catabolites generated from Nanobodies labeled using SGMIB (1 positive charge) and IB-Mal-D-GEEEK (3 negative charges) could be a factor influencing renal accumulation, as has been observed in previous studies with small peptides $(33,34)$.
Elucidating the mechanisms responsible for the excellent tumortargeting properties of *I-SGMIB-Nanobody probably will require extensive analysis of labeled catabolites generated in tumor and normal organs after in vivo administration of this labeled conjugate. Experiments to address this issue are planned; however, whatever the mechanism, the 5F7GGC-SGMIB conjugate represents a promising template for the construction of radiopharmaceuticals for the imaging, and possibly treatment, of HER2-expressing malignancies. With regard to the former, one could envision using ${ }^{123}$ I-SGMIBNanobody and ${ }^{124}$ I-SGMIB-Nanobody for SPECT and PET imaging of HER2 expression, respectively, in order to select patients and monitor response for HER2-targeted therapies. Although the 4.2-d halflife of ${ }^{124} \mathrm{I}$ might not seem ideal for this purpose, the combination of relatively prolonged tumor retention and somewhat rapid kidney excretion could be advantageous in terms of image contrast and renal radiation dose compared with shorter-lived positronemitting radionuclides such as ${ }^{18} \mathrm{~F}$ and ${ }^{68} \mathrm{Ga}$. Finally, because this ${ }^{131}$ I-SGMIB-Nanobody conjugate exhibits favorable radiation dosimetry, it might be possible to use this approach for HER2-targeted molecular radiotherapy. Moreover, a particularly appealing strategy would be to label this Nanobody with the 7.2-h $\alpha$-particle-emitting radiohalogen ${ }^{211}$ At via the SGMIB analog $N$-succinimidyl 3-211 Atastato-4-guanidino-methylbenzoate ( $\left.{ }^{211} \mathrm{At}-\mathrm{SAGMB}\right)$. Using an intact anti-EGFRvIII $\mathrm{mAb}$, the in vitro and in vivo targeting characteristics obtained with ${ }^{131} \mathrm{I}$-SGMIB and ${ }^{211}$ At-SAGMB labeling were nearly identical (40).

\section{CONCLUSION}

The results of this study demonstrate that labeling the antiHER2 Nanobody 5F7GGC using the residualizing radioiodination agent SGMIB provides a promising combination for the development of imaging agents for evaluation of HER2 expression in cancer. Radioiodination of 5F7GGC Nanobody using SGMIB resulted in a reagent with considerably improved targeting properties to BT474M1 human breast carcinoma in vitro and in vivo compared with Nanobody labeled using the IODO-GEN and IB-Mal-D-GEEEK methods. Although IB-Mal-D-GEEEK has been the optimal reagent for labeling internalizing intact mAbs, SGMIB was clearly superior for this Nanobody, underscoring the importance of matching the labeling method with the normal-tissue clearance and tumor catabolism properties of the protein molecular carrier. The high magnitude of tumor uptake and tumor-tonormal-tissue ratios observed with the 5F7GGC-125/131I-SGMIB conjugate suggest that 5 F7GGC- ${ }^{123 / 124}$ I-SGMIB could be a promising approach for imaging HER2 receptor expression.

\section{DISCLOSURE}

The costs of publication of this article were defrayed in part by the payment of page charges. Therefore, and solely to indicate this fact, this article is hereby marked "advertisement" in accordance with 18 USC section 1734. This work was supported in part by National Institutes of Health grants CA42324, CA154291, and CA14236. No other potential conflict of interest relevant to this article was reported.

\section{ACKNOWLEDGMENTS}

We thank Donna Affleck and Xiao-Guang Zhao for their excellent technical assistance in Nanobody radiolabeling and biodistribution studies. 


\section{REFERENCES}

1. Agus DB, Bunn PA, Franklin W, Garcia M, Ozols FR. HER-2/neu as a therapeutic target in non-small cell lung cancer, prostate cancer, and ovarian cancer. Semin Oncol. 2000;27:53-63.

2. Hynes NE, Lane HA. ERBB receptors and cancer: the complexity of targeted inhibitors. Nat Rev Cancer. 2005;5:341-354.

3. Hede K. Gastric cancer: trastuzumab trial results spur search for other targets. J Natl Cancer Inst. 2009;101:1306-1307.

4. Baselga J. Clinical trials of Herceptin (trastuzumab). Eur J Cancer. 2001;37 (suppl 1):18-24.

5. Agus DB, Gordon MS, Taylor C, et al. Phase I clinical study of pertuzumab, a novel HER dimerization inhibitor, in patients with advanced cancer. J Clin Oncol. 2005;23:2534-2543.

6. Blackwell KL, Burstein HJ, Storniolo AM, et al. Overall survival benefit with lapatinib in combination with trastuzumab for patients with human epidermal growth factor receptor 2-positive metastatic breast cancer: final results from the EGF104900 Study. J Clin Oncol. 2012;30:2585-2592.

7. Akabani G, Carlin S, Welsh P, Zalutsky MR. In vitro cytotoxicity of ${ }^{211}$ Atlabeled trastuzumab in human breast cancer cell lines: effect of specific activity and HER2 receptor heterogeneity on survival fraction. Nucl Med Biol. 2006;33:333-347.

8. Persson M, Gedda L, Lundqvist H, et al. $\left[{ }^{177} \mathrm{Lu}\right]$ pertuzumab: experimental therapy of HER-2-expressing xenografts. Cancer Res. 2007;67:326-331.

9. Olafsen T, Kenanova VE, Sundaresan G, et al. Optimizing radiolabeled engineered anti-p185HER2 antibody fragments for in vivo imaging. Cancer Res. 2005;65:5907-5916.

10. Löfblom J, Feldwisch J, Tolmachev V, Carlsson J, Ståhl S, Frejd FY. Affibody molecules: engineered proteins for therapeutic, diagnostic and biotechnological applications. FEBS Lett. 2010;584:2670-2680.

11. Ren G, Zhang R, Liu Z, et al. A 2-helix small protein labeled with ${ }^{68} \mathrm{Ga}$ for PET imaging of HER2 expression. J Nucl Med. 2009;50:1492-1499.

12. Vaneycken I, Devoogdt N, Van Gassen N, et al. Preclinical screening of anti-HER2 nanobodies for molecular imaging of breast cancer. FASEB J. 2011;25:2433-2446.

13. Muyldermans S, Baral TN, Cortez-Retamozzo V, et al. Camelid immunoglobulins and nanobody technology. Vet Immunol Immunopath. 2009;128:178-183.

14. Revets H, De Baetselier P, Muyldermans S. Nanobodies as novel agents for cancer therapy. Expert Opin Biol Ther. 2005;5:111-124.

15. D'Huyvetter M, Aerts A, Xavier C, et al. Development of ${ }^{177}$ Lu-nanobodies for radioimmunotherapy of HER2-positive breast cancer: evaluation of different bifunctional chelators. Contrast Media Mol Imaging. 2012;7:254-264.

16. Pruszynski M, Koumarianou E, Vaidyanathan G, et al. Targeting breast carcinoma with radioiodinated anti-HER2 nanobody. Nucl Med Biol. 2013;40:52-59.

17. Gainkam LOT, Caveliers V, Devoogdt N, et al. Localization, mechanism and reduction of renal retention of technetium-99m labeled epidermal growth factor receptor-specific nanobody in mice. Contrast Media Mol Imaging. 2011;6:85-92.

18. Vaidyanathan G, Jestin E, Olafsen T, Wu AM, Zalutsky MR. Evaluation of an anti-p185 $5^{\mathrm{HER} 2}\left(\mathrm{scFv}^{\mathrm{C}} \mathrm{C}_{\mathrm{H}} 2-\mathrm{C}_{\mathrm{H}} 3\right)_{2}$ fragment following radioiodination using two different residualizing labels: SGMIB and IB-Mal-D-GEEEK. Nucl Med Biol. 2009;36:671-680.

19. De Santes K, Slamon D, Anderson SK, et al. Radiolabeled antibody targeting of the HER-2/neu oncoprotein. Cancer Res. 1992;52:1916-1923.

20. Vaidyanathan G, Affleck DJ, Bigner DD, Zalutsky MR. Improved xenograft targeting of tumor-specific anti-epidermal growth factor receptor variant III antibody labeled using N-succinimidyl 4-guanidinomethyl-3-iodobenzoate. $\mathrm{Nucl}$ Med Biol. 2002;29:1-11.

21. Shankar S, Vaidyanathan G, Affleck DJ, Peixoto K, Bigner DD, Zalutsky MR. Evaluation of an internalizing monoclonal antibody labeled using $\mathrm{N}$-succinimidyl
3-[ $\left.{ }^{131} I\right]$ iodo-4-phosphonomethylbenzoate ([ $\left.\left.{ }^{131} \mathrm{I}\right] \mathrm{SIPMB}\right)$, a negatively charged substituent bearing acylation agent. Nucl Med Biol. 2004;31:909-919.

22. Foulon CF, Welsh PC, Bigner DD, Zalutsky MR. Positively charged templates for labeling internalizing antibodies: comparison of N-succinimidyl 5-iodo-3pyridinecarboxylate and the D-amino acid peptide KRYRR. Nucl Med Biol. 2001;28:769-777.

23. Vaidyanathan G, Alston KL, Bigner DD, Zalutsky MR. $N^{\epsilon}-(3-[* I]$ iodobenzoyl)Lys ${ }^{5}-N^{\alpha}$-maleimido-Gly ${ }^{1}$-GEEEK ([*I]IB-Mal-D-GEEEK): a radioiodinated prosthetic group containing negatively charged D-glutamates for labeling internalizing monoclonal antibodies. Bioconjug Chem. 2006;17:1085-1092.

24. Vaidyanathan G, Zalutsky MR. Synthesis of $N$-succinimidyl 4-guanidinomethyl$3-[* I]$ iodobenzoate: a radioiodination agent for labeling internalizing proteins and peptides. Nat Protoc. 2007;2:282-286.

25. Yu Z, Xia W, Wang H-Y, et al. Antitumor activity of an Ets protein, PEA3, in breast cancer cell lines MDA-MB-361DYT2 and BT474M1. Mol Carcinog. 2006;45:667-675.

26. Zahnd C, Kawe M, Stumpp MT, et al. Efficient tumor targeting with high-affinity designed ankyrin repeat proteins: effects of affinity and molecular size. Cancer Res. 2010;70:1595-1605.

27. Shankar S, Vaidyanathan G, Kuan C-T, Bigner DD, Zalutsky MR. Anti-epidermal growth factor variant III scFv fragment: effect of radioiodination method on tumor targeting and normal tissue clearance. Nucl Med Biol. 2006;33:101-110.

28. Foulon CF, Reist CJ, Bigner DD, Zalutsky MR. Radioiodination via D-amino acid peptide enhances cellular retention and tumor xenograft targeting of an internalizing anti-epidermal growth factor receptor variant III monoclonal antibody. Cancer Res. 2000;60:4453-4460.

29. Moshakis V, McIlhinney RA, Raghavan D, Neville AM. Localization of human tumour xenografts after i.v. administration of radiolabelled monoclonal antibodies. Br J Cancer. 1981;44:91-99.

30. Xavier C, Vaneycken I, D'Huyvetter M, et al. Synthesis, preclinical validation, dosimetry, and toxicity of ${ }^{68} \mathrm{Ga}$-NOTA-anti-HER2 nanobodies for iPET imaging of HER2 receptor expression in cancer. J Nucl Med. 2013;54:776-784.

31. Vosjan MJ, Perk LR, Roovers RC, et al. Facile labelling of an anti-epidermal growth factor receptor Nanobody with ${ }^{68} \mathrm{Ga}$ via a novel bifunctional desferal chelate for immuno-PET. Eur J Nucl Med Mol Imaging. 2011;38:753-763.

32. Heskamp S, Laverman P, Rosik D, et al. Imaging of human growth factor receptor type 2 expression with ${ }^{18} \mathrm{~F}$-labeled affibody molecule $\mathrm{Z}_{\text {HER2:2395 }}$ in a mouse model for ovarian cancer. J Nucl Med. 2012;53:146-153.

33. Vegt E, De Jong M, Wetzels JFM, et al. Renal toxicity of radiolabeled peptides and antibody fragments: mechanisms, impact on radionuclide therapy, and strategies for prevention. J Nucl Med. 2010;51:1049-1058.

34. Stein R, Govindan SV, Mattes MJ, et al. Improved iodine radiolabels for monoclonal antibody therapy. Cancer Res. 2003;63:111-118.

35. Tugyi R, Uray K, Iván D, Fellinger E, Perkins A, Hudecz F. Partial D-amino acid substitution: improved enzymatic stability and preserved $\mathrm{Ab}$ recognition of a MUC2 epitope peptide. Proc Natl Acad Sci USA. 2005;102:413-418.

36. Mather SJ, McKenzie AJ, Sosabowski JK, Morris TM, Ellison D, Watson SA. Selection of radiolabeled gastrin analogs for peptide receptor-targeted radionuclide therapy. J Nucl Med. 2007;48:615-622.

37. Ekblad T, Tran T, Orlova A, et al. Development and preclinical characterization of ${ }^{99 \mathrm{~m}}$ Tc-labelled Affibody molecules with reduced renal uptake. Eur J Nucl Med Mol Imaging. 2008;35:2245-2255.

38. Verrey F, Ristic Z, Romeo E, et al. Novel renal amino acid transporters. Annu Rev Physiol. 2005;67:557-572.

39. Schuldt S, Carter P, Welbourne T. Glutamate transport asymmetry and metabolism in the functioning kidney. Am J Physiol. 1999;277:E439-E446.

40. Vaidyanathan G, Affleck DJ, Bigner DD, Zalutsky MR. $N$-succinimidyl 3-[211 At] astato-4-guanidino-methylbenzoate: an acylation agent for labeling internalizing antibodies with $\alpha$-particle emitting ${ }^{211}$ At. Nucl Med Biol. 2003;30:351-359. 